\title{
Radionuclides in Sediments and Seawater at Rongelap Atoll
}

V.E. Noshkin

W.L. Robison

R.J. Eagle

J.L. Brunk

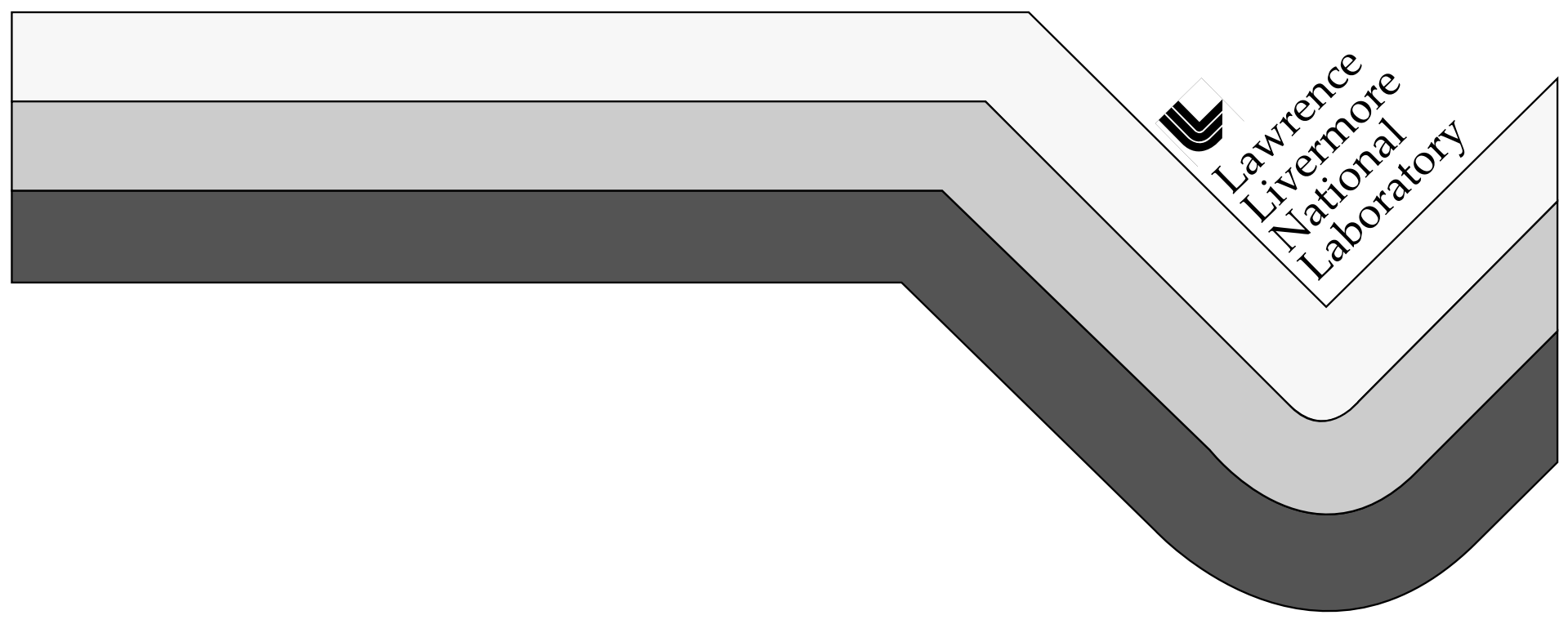




\section{DISCLAIMER}

This document was prepared as an account of work sponsored by an agency of the United States Government. Neither the United States Government nor the University of California nor any of their employees, makes any warranty, express or implied, or assumes any legal liability or responsibility for the accuracy, completeness, or usefulness of any information, apparatus, product, or process disclosed, or represents that its use would not infringe privately owned rights. Reference herein to any specific commercial product, process, or service by trade name, trademark, manufacturer, or otherwise, does not necessarily constitute or imply its endorsement, recommendation, or favoring by the United States Government or the University of California. The views and opinions of authors expressed herein do not necessarily state or reflect those of the United States Government or the University of California, and shall not be used for advertising or product endorsement purposes.

This report has been reproduced directly from the best available copy.

Available to DOE and DOE contractors from the Office of Scientific and Technical Information P.O. Box 62, Oak Ridge, TN 37831

Prices available from (423) 576-8401

Available to the public from the National Technical Information Service

U.S. Department of Commerce

5285 Port Royal Rd.,

Springfield, VA 22161

Work performed under the auspices of the U.S. Department of Energy by Lawrence Livermore National Laboratory under Contract W-7405-ENG-48. 
UCRL-LR-130250

Distribution Category UC-702

\title{
Radionuclides in Sediments and Seawater at Rongelap Atoll
}

\author{
V.E. Noshkin \\ W.L. Robison \\ R.J. Eagle \\ J.L. Brunk
}

Manuscript date: March 1998

LAWRENCE LIVERMORE NATIONAL LABORATORY 



\section{Contents}

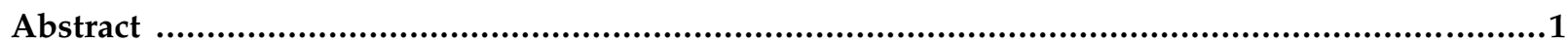

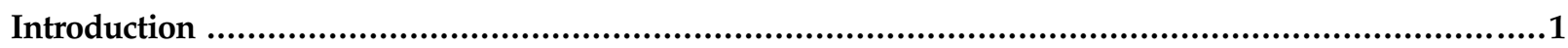

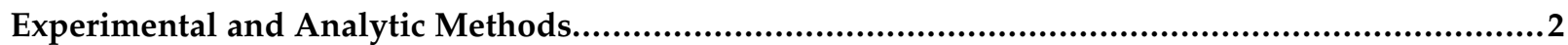

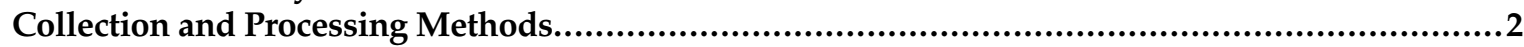

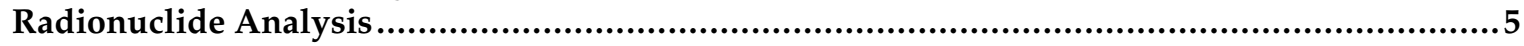

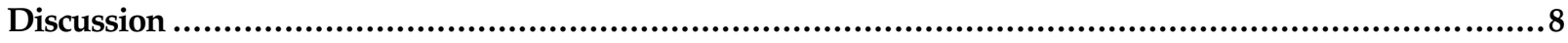

Radionuclides Associated with Sediments 24-27 Years Post Bravo........................................8

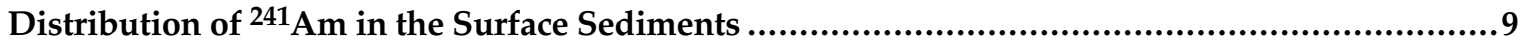

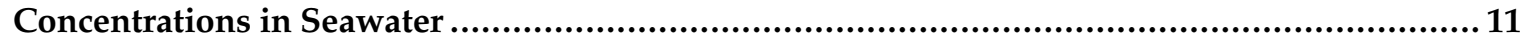

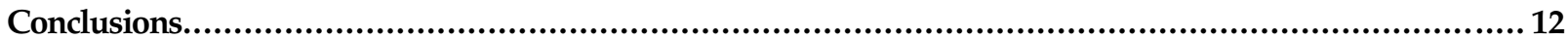

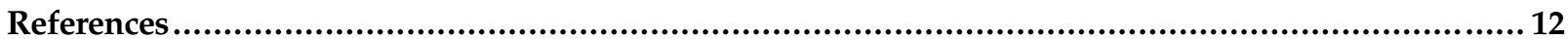





\section{List of Figures}

Figure 1. Isoconcentration plot of the inventory for ${ }^{241} \mathrm{Am}_{\left(\mathrm{kBq} \mathrm{m}^{-2}\right)}$

in the surface $(0-4 \mathrm{~cm})$ sediment from Rongelap lagoon. 10

\section{List of Tables}

Table 1. Rongelap Atoll lagoon bottom surface sediments $(0-4 \mathrm{~cm})$ collected during September 1978 and February 1981

Table 2. Concentrations of radionuclides in Rongelap surface sediments collected in September 1978

Table 3. Concentrations of gamma-emitting radionuclides in Rongelap surface sediments collected in February 1981.

Table 4. Concentration of radionuclides in surface seawater from

Rongelap Atoll during September 1978 and February 1981 ......

Table 5. Inventory and concentration of ${ }^{241} \mathrm{Am}$ and ${ }^{239+240} \mathrm{Pu}$ in surface sediments from regions of Rongelap lagoon as of February 1981. 



\title{
Radionuclides in Sediments and Seawater at Rongelap Atoll
}

\author{
Victor E. Noshkin, William L. Robison, Rodney J. Eagle, James L. Brunk
}

\begin{abstract}
The present concentrations and distributions of long-lived, man-made radionuclides in Rongelap Atoll lagoon surface sediments, based on samples collected and analyzed in 1981, are summarized in this report.

The radionuclides were associated with debris generated with the 1954 Bravo thermonuclear test at Bikini Atoll. Presently, only ${ }^{90} \mathrm{Sr}$ and the transuranic radionuclides are found associated with the surface sediments in any quantity. Other radionuclides, including ${ }^{60} \mathrm{Co}$ and ${ }^{137} \mathrm{Cs}$, are virtually absent and have either decayed or migrated from the deposits to the overlying seawater. Present inventories of ${ }^{241} \mathrm{Am}$ and ${ }^{239+240} \mathrm{Pu}$ in the surface layer at Rongelap are estimated to be $3 \%$ of the respective inventories in surface sediments from Bikini Atoll. There is a continuous slow release of the transuranics from the sediments back to the water column. The inventories will only slowly change with time unless the chemicalphysical processes that now regulate this release to the water column are changed or altered.
\end{abstract}

\section{Introduction}

The current concentrations and distributions of long-lived, man-made radionuclides in Rongelap Atoll lagoon surface sediments, based on samples collected and analyzed in 1981, are summarized in this report. Rongelap Atoll was contaminated with close-in fallout from the 15MT "Bravo" thermonuclear event that was detonated at Bikini Atoll on March 1, 1954. This test was the principal source for radioactive contamination now present in the environment. All other nuclear detonations during the 1956 Redwing and 1958 Hardtack series at the Pacific Proving Grounds contributed close-in fallout to Rongelap that amounted to a fraction of $1 \%$ of the 1954 amount, as determined from changes measured in gamma dose rates (Held, 1965). The historical events, and subsequent medical and some radiological surveillance studies associated with the fallout, the people, and the environment at Rongelap have been discussed in a number of reports and, most recently, in a series of articles which appeared in the July 1997 issue of Health Physics. These latter articles make reference to most, if not all, previous work at the atoll.

During the past 20 years, radiological surveys have focused on documenting levels of specific radionuclides in local foods, drinking water, and soils from islands of the atoll (Noshkin et al., 1981a; Robison et al., 1994; Robison and Conrado, 1996a,b; Robison et al., 1997), and in fish and invertebrates from the lagoon (Robison et al., 1981; Noshkin et al., 1981b). These surveys were undertaken to generate data to assess any potential radiological dose to present day and future inhabitants from external exposure, inhalation, and ingestion pathways.

Little information, however, is available on the man-made radionuclides in the lagoon sediments (and overlying seawater) that occupy the largest area of the atoll. Held (1963) provided a qualitative summary of early radiological data in some sediment, seawater, and other samples collected during the late 1950s. For the most part, only gross activity measurements were made on these early samples. However, in some samples collected up to 5 years after Bravo, concentrations of the fission products that included Zirconium-95 
(95 Zr), Ruthenium-106 (106 Ru), Strontium-90 $\left({ }^{90} \mathrm{Sr}\right)$, Cesium-137 ( $\left.{ }^{137} \mathrm{Cs}\right)$, Cerium-144 $\left({ }^{144} \mathrm{Ce}\right)$, Europium-155 $\left({ }^{155} \mathrm{Eu}\right)$, and Antimony-125 $\left({ }^{125} \mathrm{Sb}\right)$, and activation products that included Zinc-65 ( $\left.{ }^{65} \mathrm{Zn}\right)$, Manganese- $54\left({ }^{54} \mathrm{Mn}\right)$, Cobalt$57,60\left({ }^{57,60} \mathrm{Co}\right)$, and Iron- $55\left({ }^{55} \mathrm{Fe}\right)$, were separated and identified. The radionuclides contributing to the total activity in the sediments were associated mainly with the fine fraction (less than $0.14 \mathrm{~mm}$ ) and were concentrated in the surface 5 to $10 \mathrm{~cm}$. No manmade radionuclides were detected in seawater, but Held (1963) indicates that more sensitive analytical techniques undoubtedly would have revealed their presence.

The radionuclides in sediment and seawater do not contribute directly to human exposure. Because criteria related to human health is of primary concern, information on the fallout radioactivity in the lagoon sediments would be of secondary and lesser priority. However, Cohn et al. (1960) indicates the radionuclides are accumulated from seawater and sediments at Rongelap by local marine organisms that may eventually serve as food. Knowledge of the concentrations and distribution are required if the sediments are ever considered a resource to replace contaminated soil on islands of the atoll where they could then contribute to external exposure, and be available for uptake by indigenous plants and animals used as food. Finally, information on concentrations and distributions in sediments is of considerable scientific interest to better understand the behavior of the radionuclides within the aquatic environment of this atoll. These concerns justify an effort to learn about the radionuclides associated with the lagoon sediments and seawater.

During the 1978 Northern Marshall Island Survey (Robison et al., 1997), a few surface sediment and seawater samples were obtained for analysis from shallow depths on the lagoon reef to provide preliminary information on types and concentrations of radionuclides associated with the seawater and sediments (Noshkin et al., 1987). A more detailed sampling program was undertaken at Rongelap in 1981 to better define the distribution of persistent gamma-emitting radionuclides in the surface sediments. Lagoon seawater was again collected from selected stations in the lagoon.
The results for the transuranic and other gamma-emitting radionuclides detected in the bottom sediments and in seawater are discussed in this report.

\section{Experimental and Analytic Methods}

\section{Collection and Processing Methods}

Nearshore sediment, lagoon seawater, fish, and invertebrates were collected from Rongelap Atoll in September 1978 during the Northern Marshall Islands Radiological Survey (NMRIS). Sediment was sampled to a depth of $4 \mathrm{~cm}$ from regions of the reef near fishing sites. Overlying water depths at the sampling locations ranged from 1 to 2 meters. Information describing the samples and sampling locations is provided in Table 1. Radionuclide concentrations in parts of fish and invertebrates collected at Rongelap during the survey are discussed in Noshkin et al. (1981a), and Robison et al. (1981).

During February 1981, sediment samples were collected at stations throughout the lagoon utilizing the Marshall Island research vessel, RV Liktanur. A portable winch, mounted on the deck of the ship, was used to lower and raise a Shipek grab sampler. Sampling locations were preselected using a systematic sampling plan and treating the atoll as a square grid to ensure all regions of the lagoon were sampled. Stations were separated approximately by two nautical miles so that each sampling site was the node of a $2 \times 2$ nautical mile grid. We deviated from this grid only when the overlying water depth at a station was too shallow for the research vessel, or when the bottom material was too difficult to sample. Depths of overlying water at the stations ranged from 12 to 56 meters. Three, 5.56-cm-diameter, circular core tubes were used to subsample the contents of each grab sample to a depth of $4.0 \mathrm{~cm}$. The three samples were extruded from the cores into a single plastic bag. A total of 66 lagoon locations were sampled in this manner to generate a series of dimensionally comparative surface sediment samples. Our equipment limited us to sampling only the sediment surface. Sediments from depths deeper than $4 \mathrm{~cm}$ were not obtained. All sediment from the 1978 and 1981 programs was frozen and returned in a frozen state to LLNL for processing and analysis. At the laboratory, the 
Table 1. Rongelap Atoll lagoon bottom surface sediments $(0-4 \mathrm{~cm})$ collected during September 1978 and February 1981.

\begin{tabular}{|c|c|c|c|c|c|c|}
\hline \multirow{2}{*}{$\frac{\text { Sample ID }}{8123}$} & \multicolumn{2}{|c|}{$\begin{array}{c}\text { Collection } \\
\text { date }\end{array}$} & \multirow{2}{*}{$\begin{array}{c}\begin{array}{c}\text { Latitude } \\
\text { minutes } \\
\mathrm{N}^{\text {of }} 11^{\circ} \mathrm{N}\end{array} \\
27\end{array}$} & \multirow{2}{*}{$\begin{array}{c}\begin{array}{c}\text { Longitude } \\
\text { minutes }\end{array} \\
\text { E of } 166^{\circ} \mathrm{E}\end{array}$} & \multirow{2}{*}{$\begin{array}{c}\begin{array}{c}\text { Water } \\
\text { depth } \\
(\mathrm{m})\end{array} \\
1\end{array}$} & \multirow{2}{*}{$\begin{array}{c}\begin{array}{c}\text { Area-72.75 } \mathrm{cm}^{2} \\
\mathrm{~g} \mathrm{~cm}^{-2} \\
\text { dry wt }\end{array} \\
4.42\end{array}$} \\
\hline & $9 / 23 / 78$ & $1 \mathrm{~F}$ & & & & \\
\hline 8122 & $9 / 23 / 78$ & $7 \mathrm{~F}$ & 26 & 55 & 1 & 3.99 \\
\hline 8119 & $9 / 22 / 78$ & $9 \mathrm{~F}$ & 27 & 63.3 & 1 & 3.21 \\
\hline 8120 & $9 / 22 / 78$ & $13 \mathrm{~F}$ & 26 & 63.5 & 1 & 5.09 \\
\hline 8121 & $9 / 22 / 78$ & $23 \mathrm{~F}$ & 21.4 & 59.5 & 1 & 4.81 \\
\hline 8117 & $9 / 20 / 78$ & $33 \mathrm{~F}$ & 17 & 53.5 & 1 & 4.52 \\
\hline 8116 & $9 / 20 / 78$ & $42 \mathrm{~F}$ & 9 & 53 & 1 & 4.29 \\
\hline 8118 & $9 / 20 / 78$ & $42 \mathrm{~F}$ & 9 & 53 & 1 & 3.24 \\
\hline 8115 & $9 / 19 / 78$ & $46 \mathrm{~F}$ & 12 & 43 & 1 & 6.11 \\
\hline 8114 & $9 / 18 / 78$ & $47 \mathrm{~F}$ & 12.3 & 41.3 & 1 & 5.04 \\
\hline MSA554 & $2 / 16 / 81$ & -1 & 9.2 & 53.3 & 12 & 3.81 \\
\hline MSA555 & -2 & & 11 & 52 & 47 & 3.05 \\
\hline MSA556 & -3 & & 13 & 50 & 51 & 5.33 \\
\hline MSA557 & -4 & & 13 & 52 & 49 & 3.90 \\
\hline MSA558 & -5 & & 15 & 52 & 51 & 3.60 \\
\hline MSA559 & $2 / 17 / 81$ & -1 & 17 & 52 & 51 & 2.25 \\
\hline MSA560 & -2 & & 15 & 50 & 49 & 2.53 \\
\hline MSA561 & -3 & & 15 & 48 & 51 & 1.87 \\
\hline MSA562 & -4 & & 17 & 48 & 54 & 2.17 \\
\hline MSA563 & -5 & & 17 & 50 & 47 & 1.59 \\
\hline MSA564 & -6 & & 19 & 50 & 51 & 1.62 \\
\hline MSA565 & -7 & & 19 & 52 & 54 & 3.57 \\
\hline MSA566 & -8 & & 19 & 54 & 34 & 4.52 \\
\hline MSA567 & -9 & & 21 & 54 & 57 & 2.05 \\
\hline MSA568 & -10 & & 23 & 54 & 40 & 4.84 \\
\hline MSA569 & -11 & & 23 & 56 & 42 & 4.11 \\
\hline MSA586 & -12 & & 21 & 56 & 29 & 5.25 \\
\hline MSA587 & -13 & & 21 & 52 & 49 & 2.52 \\
\hline MSA588 & -14 & & 21 & 50 & 54 & 2.47 \\
\hline MSA589 & -15 & & 21 & 48 & 56 & 2.65 \\
\hline MSA590 & -16 & & 19 & 48 & 54 & 1.91 \\
\hline MSA591 & $2 / 18 / 81$ & -1 & 22 & 65 & 18 & 4.11 \\
\hline MSA592 & -2 & & 23 & 58 & 34 & 5.40 \\
\hline MSA593 & -3 & & 25 & 58 & 38 & 3.56 \\
\hline MSA594 & -4 & & 25 & 60 & 36 & 4.26 \\
\hline MSA595 & -5 & & 23 & 52 & 43 & 3.05 \\
\hline MSA596 & -6 & & 23 & 50 & 49 & 6.20 \\
\hline MSA597 & -7 & & 23 & 48 & 47 & 3.56 \\
\hline MSA598 & -8 & & 23 & 46 & 48 & 2.25 \\
\hline MSA599 & -9 & & 23 & 44 & 49 & 3.95 \\
\hline MSA600 & -10 & & 23 & 42 & 47 & 5.13 \\
\hline MSA611 & $2 / 19 / 81$ & -1 & 25 & 56 & 34 & 3.18 \\
\hline
\end{tabular}


Table 1. (Continued).

\begin{tabular}{|c|c|c|c|c|c|c|}
\hline \multirow{2}{*}{$\frac{\text { Sample ID }}{\text { MSA612 }}$} & \multicolumn{2}{|c|}{$\begin{array}{c}\text { Collection } \\
\text { date }\end{array}$} & \multirow{2}{*}{$\begin{array}{c}\begin{array}{c}\text { Latitude } \\
\text { minutes } \\
\mathbf{N} \text { of } 11^{\circ} \mathbf{N}\end{array} \\
25\end{array}$} & \multirow{2}{*}{$\begin{array}{c}\begin{array}{c}\text { Longitude } \\
\text { minutes } \\
\text { E of } 166^{\circ} \mathrm{E}\end{array} \\
54\end{array}$} & \multirow{2}{*}{$\begin{array}{c}\begin{array}{c}\text { Water } \\
\text { depth } \\
\text { (m) }\end{array} \\
44\end{array}$} & \multirow{2}{*}{$\begin{array}{c}\begin{array}{c}\text { Area-72.75 } \mathrm{cm}^{2} \\
\mathrm{~g} \mathrm{~cm}^{-2} \\
\text { dry wt }\end{array} \\
3.66\end{array}$} \\
\hline & -2 & & & & & \\
\hline MSA613 & -3 & & 25 & 52 & 47 & 3.55 \\
\hline MSA614 & -4 & & 27 & 52 & 34 & 1.00 \\
\hline MSA615 & -5 & & 27 & 50 & 42 & 4.08 \\
\hline MSA616 & -6 & & 25 & 50 & 48 & 3.45 \\
\hline MSA617 & -7 & & 25 & 48 & 46 & 3.31 \\
\hline MSA618 & -8 & & 25 & 46 & 51 & 2.60 \\
\hline MSA619 & -9 & & 25 & 44 & 47 & 3.75 \\
\hline MSA620 & -10 & & 25 & 42 & 46 & 4.71 \\
\hline MSA621 & -11 & & 21 & 42 & 46 & 3.22 \\
\hline MSA622 & -12 & & 21 & 44 & 54 & 5.61 \\
\hline MSA623 & -13 & & 21 & 46 & 55 & 4.82 \\
\hline MSA633 & $2 / 20 / 81$ & -1 & 19 & 46 & 51 & 1.55 \\
\hline MSA634 & -2 & & 19 & 44 & 49 & 1.53 \\
\hline MSA635 & -3 & & 19 & 42 & 46 & 0.71 \\
\hline MSA636 & -4 & & 19 & 40 & 42 & 5.40 \\
\hline MSA637 & -5 & & 17 & 40 & 42 & 5.88 \\
\hline MSA638 & -6 & & 15 & 40 & 40 & 4.00 \\
\hline MSA639 & -7 & & 13 & 40 & 29 & 5.66 \\
\hline MSA640 & -8 & & 13 & 42 & 35 & 5.69 \\
\hline MSA641 & -9 & & 13 & 44 & 40 & 5.75 \\
\hline MSA642 & -10 & & 15 & 46 & 48 & 2.21 \\
\hline MSA643 & -11 & & 15 & 44 & 49 & 5.25 \\
\hline MSA644 & -12 & & 15 & 42 & 46 & 5.14 \\
\hline MSA645 & -13 & & 17 & 42 & 47 & 3.95 \\
\hline MSA646 & -14 & & 17 & 44 & 44 & 5.61 \\
\hline MSA647 & -15 & & 17 & 46 & 55 & 1.65 \\
\hline MSA662 & $2 / 21 / 81$ & -1 & 13 & 46 & 44 & 5.29 \\
\hline MSA663 & -2 & & 13 & 48 & 48 & 5.66 \\
\hline MSA664 & -3 & & 11 & 48 & 37 & 5.11 \\
\hline MSA665 & -4 & & 10 & 48 & 35 & 5.33 \\
\hline MSA666 & -5 & & 11 & 46 & 40 & 5.42 \\
\hline MSA667 & -6 & & 11 & 50 & 42 & 5.39 \\
\hline MSA668 & -7 & & 10 & 50 & 33 & 5.54 \\
\hline
\end{tabular}


sediment samples were thawed, and the wet and dry weights were determined. The sampling locations and other relevant information for the 1981 samples are listed in Table 1.

\section{Radionuclide Analysis}

Each sample was ball-milled and transferred to containers for analysis by gamma spectrometry using $\mathrm{Ge}(\mathrm{Li})$ detection systems. Counting times were usually 1000 minutes or longer for each sample. A general-purpose computer program was used for the data reduction of all gamma-ray spectra. A brief description of the gamma-ray program and an account of our quality assurance effort are given in Noshkin et al. (1988).

Representative detection limits $\left(\mathrm{Bq} \mathrm{kg}^{-1}\right)$ for several gamma-emitting radionuclides in the 1981 samples were 1.0 for ${ }^{60} \mathrm{Co}, 0.9$ for ${ }^{137} \mathrm{Cs}$, 0.7 for Bismuth-207 $\left({ }^{207} \mathrm{Bi}\right), 3$ for ${ }^{125} \mathrm{Sb}$ and ${ }^{155} \mathrm{Eu}$, and 4 for Americium-241 $\left({ }^{241} \mathrm{Am}\right)$. All concentration data are reported on the date of collection except where noted. The radiological results for the 1978 and 1981 surface sediment samples are given in Tables 2 and 3.

Table 2. Concentrations of radionuclides in Rongelap surface sediments collected in September 1978.

\begin{tabular}{|c|c|c|c|c|c|c|c|c|}
\hline Sample ID & $\begin{array}{c}{ }^{241} \mathrm{Am}^{-1} \\
\mathrm{~Bq} \mathrm{~kg}^{-1} \\
\text { dry wt }\end{array}$ & $\begin{array}{c}{ }^{241} \mathrm{Am} \\
\mathrm{kBq} \mathrm{m^{-2 }}\end{array}$ & $\begin{array}{c}\text { Error } \\
(\%)\end{array}$ & $\begin{array}{c}{ }^{241} \mathrm{Am} / \\
{ }^{239+240} \mathrm{Pu}^{\mathrm{a}}\end{array}$ & S Value ${ }^{a, b}$ & $\begin{array}{c}{ }^{155} \mathrm{Eu}^{\mathrm{a}} \\
\mathrm{Bq} \mathrm{kg}^{-1} \\
\text { dry } \mathbf{w t}\end{array}$ & $\begin{array}{c}{ }^{137} \mathrm{Cs}^{\mathrm{a}} \\
\mathrm{Bq}^{\mathrm{kg}} \mathbf{}^{-1} \\
\text { dry wt }\end{array}$ & $\begin{array}{c}{ }^{90} \mathrm{Sr}^{\mathrm{a}} \\
\mathrm{Bq}^{\mathrm{Bg}} \mathrm{g}^{-1} \\
\text { dry wt }\end{array}$ \\
\hline 8123 & 11.0 & 0.49 & 4 & $0.63(6)$ & $0.21(30)$ & $7.4(8)$ & $1.6(18)$ & $16.0(4)$ \\
\hline 8122 & 10.8 & 0.43 & 4 & $0.68(6)$ & $0.15(40)$ & $5.6(14)$ & $<0.2$ & $29.3(4)$ \\
\hline 8119 & 5.3 & 0.17 & 18 & & & $3.2(14)$ & $<0.2$ & \\
\hline 8120 & 13.0 & 0.66 & 5 & $0.67(8)$ & $0.11(40)$ & $5.7(7)$ & $0.5(34)$ & 40.7 (3) \\
\hline 8121 & 7.5 & 0.36 & 5 & & & $5.4(7)$ & $0.6(36)$ & 21.0 \\
\hline 8117 & 3.9 & 0.17 & 7 & $0.90(8)$ & $<0.2$ & $2.6(16)$ & $<0.2$ & $2.3(14)$ \\
\hline 8116 & 3.6 & 0.15 & 5 & $0.60(8)$ & $<0.4$ & $2.4(10)$ & $<0.2$ & $4.7(8)$ \\
\hline 8118 & 3.4 & 0.11 & 6 & $0.56(8)$ & & $2.8(9)$ & 0.3 (37) & $4.1(7)$ \\
\hline 8115 & 5.6 & 0.34 & 4 & $0.61(7)$ & $<0.3$ & $3.4(9)$ & $<0.1$ & $5.1(7)$ \\
\hline \multirow[t]{3}{*}{8114} & 4.4 & 0.22 & 4 & $0.74(6)$ & $<0.4$ & $3.7(32)$ & $<0.2$ & $5.6(6)$ \\
\hline & & & mean & $0.67 \pm 0.11$ & & & & \\
\hline & & & & $0.72 \pm 0.12^{c}$ & & & & \\
\hline
\end{tabular}

a Value in parenthesis is the one sigma counting error expressed as the percentage of the value listed.

$\mathrm{b} \mathrm{S}$ is a dimensionless value and is expressed as a percentage to describe the activity of ${ }^{238} \mathrm{Pu}$ relative to the total alpha-emitting isotopes of plutonium.

$\mathrm{S}=\left({ }^{238} \mathrm{Pu}\right.$ alpha activity $) /\left({ }^{238} \mathrm{Pu}+{ }^{239} \mathrm{Pu}+{ }^{240} \mathrm{Pu}\right.$ alpha activity $\left.)\right) \times 100$.

${ }^{c}$ Estimated ratio in 1997 based on estimation of ${ }^{241} \mathrm{Pu}$ in Bikini sediment (Noshkin et al., 1997a). 
Table 3. Concentrations of gamma-emitting radionuclides in Rongelap surface sediments collected in February 1981.

\begin{tabular}{|c|c|c|c|c|c|c|c|}
\hline $\begin{array}{l}\text { MSA } \\
\text { No. }\end{array}$ & $\begin{array}{l}{ }^{241} \mathrm{Am} \\
\mathrm{Bq} \mathrm{kg}^{-1} \\
\text { dry wt }\end{array}$ & $\begin{array}{c}{ }_{\mathrm{kBq}}^{241} \mathrm{Am}^{-2} \\
\end{array}$ & $\begin{array}{c}\text { Error }^{\mathrm{a}} \\
(\%)\end{array}$ & $\begin{array}{l}{ }^{155} \mathrm{Eu}^{-1} \\
\mathrm{~Bq} \mathrm{~kg}^{-1} \\
\text { dry wt }\end{array}$ & $\begin{array}{c}{ }^{155} \mathrm{Eu} \\
\mathrm{kBq} \mathrm{m}^{-2}\end{array}$ & $\begin{array}{c}\text { Error }^{\mathrm{a}} \\
(\%)\end{array}$ & $\begin{array}{c}\text { Others detected } \\
\qquad \text { Bq kg }^{-1} \\
(\% \text { error })\end{array}$ \\
\hline 554 & 3.2 & 0.12 & 100 & 3.6 & 0.14 & 100 & \\
\hline 555 & 10.1 & 0.31 & 29 & 3.8 & 0.12 & 32 & \\
\hline 556 & 3.3 & 0.18 & 100 & 2.4 & 0.13 & 100 & \\
\hline 557 & 5.6 & 0.22 & 19 & 3.0 & 0.12 & 34 & \\
\hline 558 & 4.1 & 0.15 & 100 & 2.6 & 0.09 & 100 & \\
\hline 559 & 32.7 & 0.74 & 12 & 11.5 & 0.26 & 16 & \\
\hline 560 & 21.5 & 0.54 & 16 & 6.1 & 0.16 & 22 & \\
\hline 561 & 18.6 & 0.35 & 29 & 3.0 & 0.06 & 100 & \\
\hline 562 & 13.6 & 0.30 & 27 & 4.2 & 0.09 & 53 & \\
\hline 563 & 13.9 & 0.22 & 15 & 4.9 & 0.08 & 32 & \\
\hline 564 & 25.9 & 0.42 & 16 & 1.5 & 0.02 & 100 & \\
\hline 565 & 12.5 & 0.45 & 11 & 4.1 & 0.15 & 24 & \\
\hline 566 & 3.7 & 0.17 & 100 & 2.6 & 0.12 & 100 & \\
\hline 567 & 20.0 & 0.41 & 16 & 6.5 & 0.13 & 28 & \\
\hline 568 & 16.6 & 0.80 & 23 & 4.6 & 0.22 & 38 & ${ }^{60} \mathrm{Co}-1.7(57)$ \\
\hline 569 & 9.7 & 0.40 & 32 & 7.7 & 0.32 & 30 & \\
\hline 586 & 4.4 & 0.23 & 100 & 3.0 & 0.16 & 100 & \\
\hline 587 & 24.8 & 0.62 & 14 & 6.7 & 0.17 & 31 & \\
\hline 588 & 29.8 & 0.74 & 12 & 8.4 & 0.21 & 18 & \\
\hline 589 & 21.1 & 0.56 & 23 & 10.7 & 0.28 & 19 & \\
\hline 590 & 23.3 & 0.45 & 19 & 6.8 & 0.13 & 38 & ${ }^{40} \mathrm{~K}-42(39)$ \\
\hline 591 & 8.9 & 0.37 & 22 & 4.2 & 0.17 & 38 & \\
\hline 592 & 3.7 & 0.20 & 100 & 2.6 & 0.14 & 100 & \\
\hline 593 & 26.3 & 0.94 & 10 & 5.9 & 0.21 & 22 & \\
\hline 594 & 15.0 & 0.64 & 24 & 3.0 & 0.13 & 100 & \\
\hline 595 & 41.5 & 1.27 & 9 & 15.9 & 0.49 & 13 & \\
\hline 596 & 23.3 & 1.45 & 18 & 5.9 & 0.37 & 29 & \\
\hline 597 & 30.4 & 1.08 & 16 & 12.6 & 0.45 & 16 & ${ }^{137} \mathrm{Cs}-1.4(38) ;{ }^{40} \mathrm{~K}-21(31)$ \\
\hline 598 & 41.1 & 0.93 & 14 & 12.6 & 0.28 & 17 & ${ }^{137} \mathrm{Cs}-2.4(38) ;{ }^{40} \mathrm{~K}-37(36)$ \\
\hline 599 & 25.6 & 1.01 & 12 & 8.7 & 0.34 & 14 & \\
\hline 600 & 21.5 & 1.10 & 18 & 8.4 & 0.43 & 18 & \\
\hline 611 & 17.1 & 0.54 & 21 & 3.3 & 0.11 & 100 & \\
\hline 612 & 23.6 & 0.86 & 14 & 9.0 & 0.33 & 18 & \\
\hline 613 & 15.2 & 0.54 & 25 & 3.0 & 0.11 & 100 & \\
\hline 614 & 32.6 & 0.33 & 20 & 8.7 & 0.09 & 47 & \\
\hline 615 & 24.3 & 0.99 & 10 & 9.7 & 0.40 & 15 & \\
\hline 616 & 38.1 & 1.32 & 13 & 14.1 & 0.49 & 12 & ${ }^{137} \mathrm{Cs}-2.3(25)$ \\
\hline 617 & 32.8 & 1.09 & 6 & 12.0 & 0.40 & 11 & ${ }^{137} \mathrm{Cs}-1.7(41)$ \\
\hline 618 & 47.5 & 1.23 & 17 & 17.5 & 0.45 & 15 & ${ }^{137} \mathrm{Cs}-2.6(28)$ \\
\hline
\end{tabular}


Table 3. (Continued).

\begin{tabular}{|c|c|c|c|c|c|c|c|}
\hline $\begin{array}{c}\text { MSA } \\
\text { No. }\end{array}$ & $\begin{array}{l}{ }^{241} \mathrm{Am} \\
\mathrm{Bq} \mathrm{kg}^{-1} \\
\text { dry wt }\end{array}$ & $\begin{array}{c}{ }^{241} \mathrm{Am} \\
\mathrm{kBq} \mathrm{m}^{-2}\end{array}$ & $\begin{array}{c}\text { Error }^{\mathrm{a}} \\
(\%)\end{array}$ & $\begin{array}{l}{ }^{155} \mathrm{Eu}^{-1} \\
\mathrm{~Bq}^{\mathrm{kg}} \mathbf{1}^{-1} \\
\text { dry wt }\end{array}$ & $\begin{array}{c}{ }^{155} \mathrm{Eu} \\
\mathrm{kBq} \mathrm{m}^{-2}\end{array}$ & $\begin{array}{c}\text { Error }^{\mathrm{a}} \\
(\%)\end{array}$ & $\begin{array}{l}\text { Others detected }{ }^{b} \\
\text { Bq kg }^{-1} \\
(\% \text { error })\end{array}$ \\
\hline 619 & 33.1 & 1.24 & 10 & 9.4 & 0.35 & 16 & \\
\hline 620 & 41.5 & 1.96 & 15 & 14.0 & 0.66 & 18 & ${ }^{137} \mathrm{Cs}-2.5(37)$ \\
\hline 621 & 15.8 & 0.51 & 17 & 8.8 & 0.28 & 23 & \\
\hline 622 & 25.5 & 1.43 & 17 & 8.3 & 0.47 & 17 & ${ }^{40} \mathrm{~K}-17(31)$ \\
\hline 623 & 28.3 & 1.37 & 7 & 9.3 & 0.45 & 13 & \\
\hline 633 & 27.7 & 0.43 & 15 & 9.6 & 0.15 & 26 & \\
\hline 634 & 24.2 & 0.37 & 22 & 8.2 & 0.13 & 25 & \\
\hline 635 & 28.4 & 0.20 & 8 & 10.7 & 0.08 & 17 & \\
\hline 636 & 10.7 & 0.58 & 35 & 2.8 & 0.15 & 100 & \\
\hline 637 & 13.2 & 0.78 & 26 & 4.1 & 0.24 & 36 & \\
\hline 638 & 7.1 & 0.29 & 40 & 4.6 & 0.18 & 28 & \\
\hline 639 & 3.1 & 0.17 & 100 & 3.5 & 0.20 & 100 & \\
\hline 640 & 2.9 & 0.16 & 100 & 2.1 & 0.12 & 100 & \\
\hline 641 & 7.3 & 0.42 & 22 & 3.4 & 0.20 & 32 & \\
\hline 642 & 16.8 & 0.37 & 21 & 3.9 & 0.09 & 51 & \\
\hline 643 & 13.0 & 0.68 & 25 & 4.6 & 0.24 & 32 & \\
\hline 644 & 4.9 & 0.25 & 100 & 3.4 & 0.18 & 100 & \\
\hline 645 & 15.6 & 0.62 & 20 & 3.5 & 0.14 & 100 & ${ }^{137} \mathrm{Cs}-2.8(23) ;{ }^{40} \mathrm{~K}-15(40)$ \\
\hline 646 & 7.2 & 0.40 & 36 & 2.6 & 0.14 & 100 & \\
\hline 647 & 17.6 & 0.29 & 12 & 4.7 & 0.08 & 22 & \\
\hline 662 & 4.1 & 0.22 & 100 & 3.1 & 0.16 & 100 & \\
\hline 663 & 5.3 & 0.30 & 100 & 3.6 & 0.20 & 100 & \\
\hline 664 & 3.5 & 0.18 & 100 & 3.9 & 0.20 & 100 & \\
\hline 665 & 2.5 & 0.13 & 100 & 2.1 & 0.11 & 100 & \\
\hline 666 & 3.6 & 0.20 & 100 & 4.4 & 0.24 & 38 & ${ }^{137} \mathrm{Cs}-2.0(33)$ \\
\hline 667 & 4.7 & 0.25 & 26 & 3.1 & 0.17 & 52 & \\
\hline 668 & 3.8 & 0.21 & 100 & 2.7 & 0.15 & 100 & \\
\hline
\end{tabular}

a Error(\%)—one sigma counting error expressed as the percentage of the value listed.

${ }^{b}$ Except where indicated, ${ }^{60}$ Co was below detection limits of $1.0 \pm 0.5 \mathrm{~Bq} \mathrm{~kg}^{-1}$ in remaining samples.

$\mathrm{b}$ Except where indicated, ${ }^{137} \mathrm{Cs}$ was below detection limits of $0.9 \pm 0.3 \mathrm{~Bq} \mathrm{~kg}^{-1}$ in remaining samples. b ${ }^{207} \mathrm{Bi}$ was below detection limits of $0.7 \pm 0.3 \mathrm{~Bq} \mathrm{~kg}^{-1}$ in all samples.

The concentrations of Plutonium-239+240 $(239+240 \mathrm{Pu})$ and ${ }^{90} \mathrm{Sr}$ were determined in the 1978 samples following chemical separation procedures described in Wong et al. (1994). Plutonium-239+240 and ${ }^{90}$ Sr were not separated from the 1981 samples. Several radionuclides were separated and measured in the lagoon seawater samples collected during 1978 and 1981. Water samples were first filtered through an in-line filter. The filter used to remove particulates from the samples is a $1-\mu \mathrm{m}$ Micro-Wyndell@, DDCCPY filter cartridge (AMF, Cuno Division, Meriden, Connecticut). We have demonstrated that this filter is as efficient in removing suspended particulates from the lagoon water as a 0.2 to $0.3-\mu \mathrm{m}$ filter (Wong et al., 1980). Therefore, solution concentrations shown in Table 4 refer to 
Table 4. Concentration of radionuclides in surface seawater from Rongelap Atoll during September 1978 and February 1981.

\begin{tabular}{|c|c|c|c|c|c|c|c|c|c|c|c|c|c|c|}
\hline $\begin{array}{l}\text { Island } \\
\text { ID }\end{array}$ & $\begin{array}{c}\text { Latitude } \\
\text { minutes } \\
\mathrm{N} \text { of } 11^{\circ} \mathrm{N}\end{array}$ & $\begin{array}{c}\text { Longitude } \\
\text { minutes } \\
\mathrm{E} \text { of } 166^{\circ} \mathrm{E}\end{array}$ & $\begin{array}{c}{ }^{239+240} \mathrm{Pu} \\
\text { solution } \\
\mathrm{mBq}^{-1}\end{array}$ & $\underset{(\%)}{\text { Error }^{a}}$ & $\begin{array}{l}{ }^{239+240} \mathrm{Pu} \\
\text { prefilter } \\
\mathrm{mBq}^{-1}\end{array}$ & $\underset{(\%)}{\text { Error }^{a}}$ & $\begin{array}{l}{ }^{241} \mathrm{Am} \\
\text { solution } \\
\mathrm{mBq}^{-1}\end{array}$ & $\begin{array}{l}\text { Error }^{a} \\
(\%)\end{array}$ & $\begin{array}{l}{ }_{\text {prefilter }}^{241} \mathrm{Am} \\
\mathrm{mBq}^{-1}\end{array}$ & $\begin{array}{c}\text { Error }^{\mathrm{a}} \\
(\%)\end{array}$ & $\begin{array}{c}{ }^{137} \mathrm{Cs} \\
\mathrm{mBq}^{-1}\end{array}$ & $\begin{array}{c}\text { Error }^{a} \\
(\%)\end{array}$ & $\begin{array}{c}{ }^{90} \mathrm{Sr} \\
\mathrm{mBq}^{-1}\end{array}$ & $\begin{array}{c}\text { Error }^{\mathrm{a}} \\
(\%)\end{array}$ \\
\hline \multicolumn{15}{|c|}{ Rongelap Atoll (September 1978) } \\
\hline $\mathrm{F}-1$ & 27 & 41.5 & 57 & 12 & & & 12 & 28 & 10 & 26 & 4.96 & 3 & 4.11 & 6 \\
\hline F-7 & 26 & 55 & 42 & 22 & & & 29 & 32 & 7 & 39 & 5.00 & 3 & 4.11 & 10 \\
\hline F-9 & 27 & 63.3 & 39 & 14 & 106 & 5 & & & & & 4.96 & 3 & & \\
\hline F-23 & 21.4 & 59.5 & 46 & 22 & 26 & 17 & 11 & 50 & 18 & 13 & 6.96 & 3 & 4.37 & 4 \\
\hline F-33 & 17 & 53.5 & 64 & 11 & 4 & 36 & 28 & 34 & 4 & 57 & 5.70 & 3 & 3.67 & 7 \\
\hline $\mathrm{F}-47$ & 12.3 & $\begin{array}{l}41.3 \\
\text { Mean }\end{array}$ & $\begin{array}{c}42 \\
48 \pm 10\end{array}$ & 31 & 62 & 24 & 7 & 100 & 11 & 50 & $\begin{array}{c}4.78 \\
5.4 \pm 0.8\end{array}$ & 7 & $\begin{array}{c}4.15 \\
4.1 \pm 0.3\end{array}$ & 11 \\
\hline \multicolumn{15}{|c|}{ Rongelap (February 1981) } \\
\hline D758 & 13 & 50 & 52 & 8 & & & & & & & & & & \\
\hline D759 & 9 & 52 & 44 & 9 & & & & & & & & & & \\
\hline D760 & 19 & 50 & 41 & 13 & & & & & & & & & & \\
\hline D761 & 17 & 53.3 & 81 & 7 & & & & & & & & & & \\
\hline D762 & 26 & 32 & 30 & 12 & & & & & & & & & & \\
\hline D763 & 26 & 55 & 25 & 16 & & & & & & & & & & \\
\hline D764 & 25 & 56 & 20 & 14 & & & & & & & & & & \\
\hline D765 & 19 & 40 & 33 & 12 & & & & & & & & & & \\
\hline D766 & 13 & 48 & 41 & 12 & & & & & & & & & & \\
\hline D767 & 26.7 & 41 & 44 & 12 & & & & & & & & & & \\
\hline D768 & 10 & $\begin{array}{l}50 \\
\text { mean }\end{array}$ & $\begin{array}{l}41 \\
41 \pm 16\end{array}$ & 10 & & & & & & & & & & \\
\hline \multicolumn{15}{|c|}{$\begin{array}{l}\text { Mean concentration in } \\
\text { north equatorial } \\
\text { Pacific surface water }\end{array}$} \\
\hline 1972- & -1984 & & 15 & & & & & & & & $5.2 \pm 0.4$ & & & \\
\hline
\end{tabular}

a Error (\%)—one sigma counting error expressed as the percentage of value listed.

the quantity passing through a $1-\mu \mathrm{m}$ filter and prefilter samples refer to the particulate phase associated with the $1-\mu \mathrm{m}$ filter.

\section{Discussion}

\section{Radionuclides Associated with Sediments 24-27 Years Post Bravo}

The data in Tables 2 and 3 show that the man-made gamma-emitting radionuclides found above detection limits in most 1978 and 1981 samples included only ${ }^{241} \mathrm{Am}$ and ${ }^{155} \mathrm{Eu}$. By $1981,{ }^{137}$ Cs was identified in only 8 of the 66 samples at a level no greater than $3 \mathrm{~Bq} \mathrm{~kg}^{-1}$ and ${ }^{60} \mathrm{Co}$ was detected in only one sample. In a few samples, naturally occurring Potassium- 40 $\left({ }^{40} \mathrm{~K}\right)$, and daughter products in the uranium decay series (concentrations not provided in the tables) were the only other gamma-emitting radionuclides detected with the $\mathrm{Ge}(\mathrm{Li})$ systems. Other man-made gamma-emitting radionuclides, such as ${ }^{207} \mathrm{Bi},{ }^{152,154} \mathrm{Eu},{ }^{125} \mathrm{Sb}$, and Rhodium-101,102m (101,102m Rh), measured in some sediment samples during the same period from Bikini lagoon (Noshkin et al., 1997b), were below detection limits in all Rongelap samples. Other man-made, gammaemitting radionuclides, that were shown by Held (1963) to be associated with the sediments in the late 1950s have been reduced 
to levels that are below detection limits in 100300-g samples.

In 1981, the mean ${ }^{155} \mathrm{Eu}$ to ${ }^{241} \mathrm{Am}$ activity ratio in surface sediments, computed from the data in Table 3, was $0.37 \pm 0.12$. By 1997, the ratio is reduced to at least $0.040 \pm 0.012$ due to loss of ${ }^{155} \mathrm{Eu}$ by radioactive decay. It will essentially be below detection limits in any surface sediment sample collected after 1997. Of the many gamma-emitting radionuclides originally deposited in the lagoon with fallout debris, only ${ }^{241} \mathrm{Am}$ will be measurable at concentrations not exceeding $50 \mathrm{~Bq} \mathrm{~kg}^{-1}$ dry weight in lagoon surface sediments through the year 2000. In island soils, however, mean levels of ${ }^{137} \mathrm{Cs}$ presently exceed those of ${ }^{241} \mathrm{Am}$ (Robison and Conrado, 1996a,b). Therefore, ${ }^{137} \mathrm{Cs}$ will be measurable through the year 2000 , but only in association with materials originating from the terrestrial environment.

In 1978 , the mean ${ }^{241} \mathrm{Am}$ to ${ }^{239+240} \mathrm{Pu}$ activity ratio in the surface sediments from eight different locations was $0.67 \pm 0.11$. This value is assumed to be representative of the ratio in sediments from anywhere within the atoll. It is in excellent agreement with the mean value of $0.69 \pm 0.17$ determined in 19 surface sediment samples collected at Bikini Atoll in 1979 (Noshkin et al., 1997a). The ratio will increase slightly to a value of $0.72 \pm 0.12$ by 1997 from growth of ${ }^{241} \mathrm{Am}$ based on estimated residual quantities of the parent radionuclide, ${ }^{241} \mathrm{Pu}$, in the environment (Hisamatsu and Sakanoue, 1978; Noshkin et al., 1997a). Therefore, ${ }^{239+240} \mathrm{Pu}$ will be measurable in the sediments through the year 2000 at levels somewhat higher than ${ }^{241} \mathrm{Am}$.

The $S$ value, which describes the activity of ${ }^{238} \mathrm{Pu}$ relative to the total alpha-emitting isotopes $\left({ }^{238} \mathrm{Pu}+{ }^{239} \mathrm{Pu}+{ }^{240} \mathrm{Pu}\right)$ as a percent, was computed in only three samples where ${ }^{238} \mathrm{Pu}$ was above detection limits. These three samples were obtained from the northern half of the lagoon. The mean $S$ value associated with the samples is $0.16 \pm 0.05$. This is comparable to an $S$ value of $0.10 \pm 0.01$, determined at this laboratory, in "Bravo" test debris deposited on the fishing boat, FukuryuMaru. The boat was located east of Bikini and to the north of Rongelap atoll at 166 degrees 58 minutes N, 11 degrees 53 minutes E on March 1, 1954, and was contaminated with fallout debris from the Bravo test. The similar mean $S$ value associated with the fallout debris from the ship and in the sediment from the atoll indicates, as expected, a common source for this radioactivity. In 1997, the $S$ value will be reduced slightly to 0.09 due to decay of ${ }^{238} \mathrm{Pu}$. Concentrations of ${ }^{238} \mathrm{Pu}$ in this environment are of little radiological significance.

Strontium-90 was also detected in the 1978 lagoon sediment samples following radiochemical separation and analysis of its daughter radionuclide, Yttrium-90 $\left({ }^{90} \mathrm{Y}\right)$, by beta counting. This radionuclide is strongly associated with carbonate sediments and will slowly decrease in the surface sediments primarily by radioactive decay. Unlike ${ }^{155} \mathrm{Eu}$ and ${ }^{239+240} \mathrm{Pu},{ }^{90} \mathrm{Sr}$ and ${ }^{241} \mathrm{Am}$ are not strongly correlated in the sediments. Concentration ratios of ${ }^{241} \mathrm{Am}$ to ${ }^{90} \mathrm{Sr}$ are found to range from 0.3 to 1.7 , respectively.

Neither ${ }^{241} \mathrm{Am},{ }^{239+240} \mathrm{Pu}$, nor ${ }^{90} \mathrm{Sr}$ are significantly concentrated in edible parts of marine organisms. These radionuclides in any marine food chain will continue to contribute only a small fraction of the total effective dose to individuals at this atoll (Robison, et al., 1981; Robison et al., 1994).

\section{Distribution of ${ }^{241} \mathrm{Am}$ in the Surface Sediments}

The surface sediment inventory values for ${ }^{241} \mathrm{Am}$ were plotted on a lagoon chart. Isoconcentration lines were constructed to distinguish regions of the bottom surface sediment with comparable values. The data provided in Table 3 were used to construct the spatial distribution shown in Figure 1. Highest inventories are associated with the sediment from the northern half of the atoll. Gradients decrease in a southerly direction to regions near the South Pass where ${ }^{241} \mathrm{Am}$ is below detection in the surface sediments. The different isopleths roughly coincide with the estimated fallout path of differently contaminated debris from the 1954 Bravo event (Robison et al., 1997). There is approximately a factor of 10 difference in concentration associated with surface sediments from the north compared to samples from the southern part of the atoll. This was also the difference estimated for the gamma dose rates on northern and southern islands one day after the Bravo test (Held, 1965). Following deposition to the lagoon surface water, some fraction of the particles contaminated with ${ }^{241} \mathrm{Am}$ (or its parent, ${ }^{241} \mathrm{Pu}$ ) 


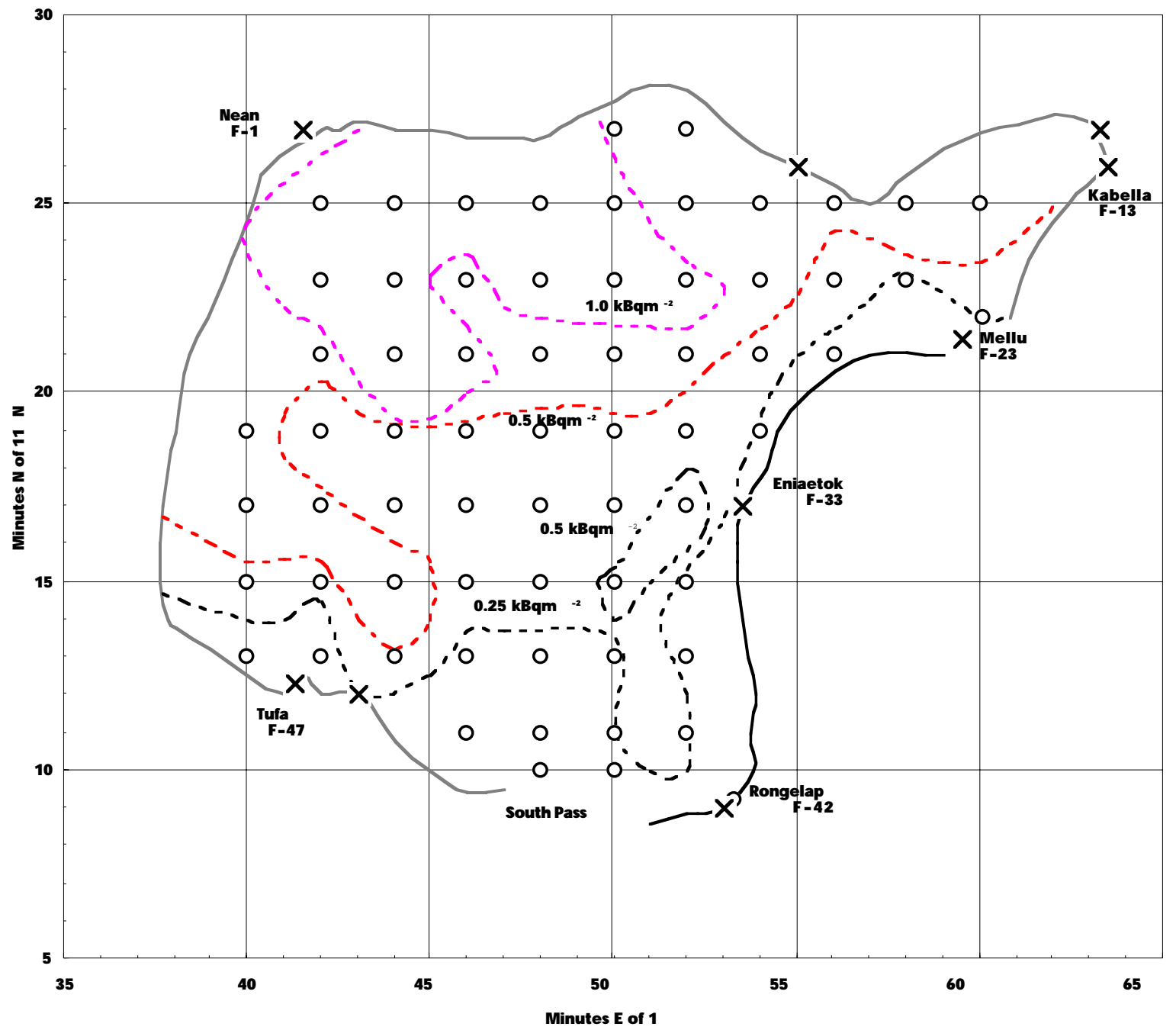

Figure 1. Isoconcentration plot of the inventory for ${ }^{241} \mathrm{Am}\left(\mathrm{kBq} \mathrm{m}^{-2}\right)$ in the surface $(0-4 \mathrm{~cm})$ sediment from Rongelap lagoon.

settled rapidly to the bottom sediment, which accounts for the current distribution pattern that resembles the original 1954 fallout pattern over the atoll.

In Figure 1, the area between the contour intervals was determined along with the lognormal mean inventory $\left(\mathrm{kBq} \mathrm{m}^{-2}\right)$ within the respective regions. Multiplying the area by the mean inventory provides an estimate of the total amount of ${ }^{241} \mathrm{Am}$ associated with the sediment within the region. Summing these regional amounts generates a value for the total amount associated with the surface

4-cm layer of sediment over the entire lagoon. The mean activity ratio for ${ }^{241} \mathrm{Am}$ to ${ }^{239+240} \mathrm{Pu}$ of $0.67 \pm$ 0.11 is used to estimate the amount of ${ }^{239+240} \mathrm{Pu}$ associated with the surface sediments. Respective areas and inventory values are shown in Table 5 . The mean concentrations $\left(\mathrm{Bq} \mathrm{kg}^{-1}\right)$ within the inventory intervals are also calculated and shown in Table 5 for information. The total quantities of ${ }^{241} \mathrm{Am}$ and ${ }^{239+240} \mathrm{Pu}$ in the lagoon-sediment surface (4-cm deep) of are $0.63 \pm 0.09$ and $0.94 \pm 0.16 \mathrm{TBq}$, 
Table 5. Inventory and concentration of ${ }^{241} \mathrm{Am}$ and ${ }^{239+240} \mathrm{Pu}$ in surface sediments from regions of Rongelap lagoon as of February 1981. Area of Rongelap lagoon-1025 km².

\begin{tabular}{|c|c|c|c|c|}
\hline $\begin{array}{l}{ }^{241} \mathrm{Am} \text { Inventory } \\
\text { interval }\left(\mathrm{kBq} \mathrm{m}^{-2}\right)\end{array}$ & $\begin{array}{l}\text { Mean inventory } \\
\left(\mathrm{kBq} \mathrm{m}^{-2}\right)\end{array}$ & $\begin{array}{c}{ }^{241} \mathrm{Am} \\
\text { Mean concentration } \\
\left(\mathrm{Bq} \mathrm{kg}^{-1}\right)\end{array}$ & $\begin{array}{c}\text { Area of interval } \\
\qquad\left(\mathrm{km}^{2}\right)\end{array}$ & $\begin{array}{l}\text { TBq in interval } \\
\text { to depth of } 4 \mathrm{~cm}\end{array}$ \\
\hline $\begin{array}{l}>1 \\
0.5-1 \\
0.25-0.5 \\
0-0.25\end{array}$ & $\begin{array}{l}1.3 \pm 0.3 \\
0.7 \pm 0.2 \\
0.36 \pm 0.10 \\
0.19 \pm 0.03\end{array}$ & $\begin{array}{c}31 \pm 10 \\
20 \pm 9 \\
16 \pm 8 \\
5 \pm 2\end{array}$ & $\begin{array}{c}204 \\
289 \\
333 \\
199 \\
\text { Lagoon total }\end{array}$ & $\begin{array}{l}0.27 \pm 0.06 \\
0.20 \pm 0.06 \\
0.12 \pm 0.03 \\
\underline{0.04 \pm 0.01} \\
0.63 \pm 0.09\end{array}$ \\
\hline $\begin{array}{l}\text { Estimated } \\
{ }^{241} \mathrm{Am} \text { inventory } \\
\text { interval }\left(\mathrm{kBq} \mathrm{m}^{-2}\right)\end{array}$ & ventory of $239+240$ & a in surface sediments & om regions of Rong & $\begin{array}{l}\text { p lagoon } \\
\text { lepth of } 4.0 \mathrm{~cm}^{\mathrm{a}}\end{array}$ \\
\hline $\begin{array}{l}>1 \\
0.5-1 \\
0.25-0.5 \\
0-0.25\end{array}$ & & & Lagoon total & $\begin{array}{l}0.40 \pm 0.11 \\
0.30 \pm 0.10 \\
0.18 \pm 0.05 \\
\underline{0.06 \pm 0.02} \\
0.94 \pm 0.16\end{array}$ \\
\hline
\end{tabular}

a ${ }^{241} \mathrm{Am}$-inventory value divided by $0.67 \pm 0.11$.

respectively. These quantities represent approximately $3 \%$ of the respective inventories of ${ }^{241} \mathrm{Am}$ and ${ }^{239+240} \mathrm{Pu}$ associated with the surface sediment $(4 \mathrm{~cm}$ deep) at Bikini Atoll (Noshkin et al., 1997a). The highest levels of ${ }^{241} \mathrm{Am}$ in surface sediments found in the NW quadrant of Rongelap are equivalent in value to the lowest levels in surface material found near the East Channel of Bikini lagoon. Unlike Bikini, the surface sediments from anywhere within Rongelap lagoon contain essentially no ${ }^{137} \mathrm{Cs}$ and low levels of the transuranic radionuclides. The sediments are less contaminated than the soil from the islands, and consequently, provide a better carbonate material if replacement of contaminated island soil is required at either Bikini or Rongelap Atolls. These estimated inventories must be viewed as lower limits since the radionuclides are no doubt present in material from greater depths in the sediment column. At Bikini, for example, only $28 \pm 10 \%$ of the ${ }^{241} \mathrm{Am}$ is associated with the surface $4 \mathrm{~cm}$ of sediment, and recall that Held (1965) indicates that early sediment samples contained radioactivity to at least a depth of $10 \mathrm{~cm}$. At this time, it is not possible to speculate on the fraction of the total inventory represented by the amount in the surface $4 \mathrm{~cm}$ layer of sediment.

\section{Concentrations in Seawater}

The data in Table 4 shows that the concentrations of ${ }^{239+240} \mathrm{Pu}$ in all lagoon water collected in 1978 and 1981 exceed the background levels of $15 \pm 7 \mu \mathrm{Bq} \mathrm{l}^{-1}$ measured in the equatorial Pacific surface waters between 1972 and 1982 (Noshkin et al, 1987). The comparable mean amounts of ${ }^{239+240} \mathrm{Pu}$ found in solution during both years shows that these higher than oceanic background levels are real and persistent. Mobilization of small amounts of ${ }^{239+240} \mathrm{Pu}$ (and ${ }^{241} \mathrm{Am}$ ) from sediments to the overlying bottom water is responsible for the elevated levels in the seawater within Rongelap lagoon. A comparable process adds 
excess transuranic radionuclides to the water column at Bikini and Enewetak (Noshkin, 1980; Noshkin and Wong, 1980; Noshkin et al., 1987).

Data in Table 4 show that the average concentration of ${ }^{137} \mathrm{Cs}$ in surface equatorial seawater between 1967 and 1982 was $5.2 \pm 0.4$ $\mathrm{mBq}^{-1}$ (Noshkin et al., 1987). Unlike the ${ }^{239+240} \mathrm{Pu}$ and ${ }^{241} \mathrm{Am}$ concentrations, the ${ }^{137} \mathrm{Cs}$ levels in the lagoon seawater are indistinguishable from fallout levels in the equatorial Pacific Ocean. Essentially the entire inventory of ${ }^{137} \mathrm{Cs}$ associated with the local fallout that reached the lagoon sediments in 1954 has mobilized to the overlying seawater. This labeled water is eventually mixed with surface water that is then transported by prevailing surface currents out of the lagoon to the surface water of the north equatorial Pacific ocean.

Concentrations of ${ }^{90} \mathrm{Sr}$ in the lagoon water are not significantly different from the global fallout concentrations in lagoon seawater at less contaminated atolls such as Ujelang, Likiep, and Wotho (Noshkin et al., 1987). Therefore, unlike ${ }^{239+240} \mathrm{Pu}$ and ${ }^{241} \mathrm{Am}$, any ${ }^{90} \mathrm{Sr}$ mobilized from sediments to seawater is masked by the throughput of global fallout concentrations associated with the north equatorial surface water, which continuously exchanges with the lagoon water mass.

\section{Conclusions}

The lagoon sediments at Rongelap Atoll were contaminated with a variety of fission and activation products from settling fallout particles generated by the Bravo thermonuclear test held at Bikini Atoll in 1954. Other tests conducted during 1956 and 1958 at Bikini and Enewetak added less than a fraction of $1 \%$ of that from the Bravo event to the closein fallout at Rongelap. By 1997, the only longlived radionuclides found in any quantity associated with lagoon surface sediments at Rongelap Atoll would be ${ }^{90} \mathrm{Sr}$ and the transuranic radionuclides, ${ }^{241} \mathrm{Am}$ and ${ }^{239+240} \mathrm{Pu}$. These radionuclides are not significantly concentrated in edible parts of marine organisms, and will continue to contribute only a small fraction of the total effective dose to individuals at this atoll from the marine ingestion pathway. Highest amounts of ${ }^{241} \mathrm{Am}$ are associated with bottom sediments from the northern half of the atoll. Concentration gradients decrease in a southerly direction to regions near the South Pass where ${ }^{241} \mathrm{Am}$ is below limits of detection. Little postdepositional migration of particles with ${ }^{241} \mathrm{Am}$ contamination appears to have occurred since the fallout material fell to the bottom of the lagoon in 1954. The inventories of ${ }^{241} \mathrm{Am}$ and ${ }^{239+240} \mathrm{Pu}$ in the surface $4 \mathrm{~cm}$ of sediment are approximately $3 \%$ of the quantity in surface sediments from Bikini Atoll. Since the sediments contain no measurably high levels of other gamma emitters and low levels of the transuranic radionuclides, they are radiologically better materials if sediments are ever required to replace contaminated soil on either Bikini or Rongelap Atolls. There is a continuous low release of ${ }^{239+240} \mathrm{Pu}$ and ${ }^{241} \mathrm{Am}$ from the sediments back to the water column. The inventories will change only slowly with time unless the chemical/physical processes that now regulate this release to the water column are changed or altered.

\section{References}

Cohn, S. H., J. S. Robertson, and R.A. Conard (1960), "Radioisotopes and Environmental Circumstances: The Internal Radioactive Contamination of a Pacific Island Community Exposed to Local Fallout," in Radioisotopes in the Biosphere, Caldecott, R. S., and Snyder, L. A., Eds., University of Minnesota Printing Department, Minneapolis, MN.

Held, E. E. (1963), "Qualitative Distribution of Radionuclides at Rongelap Atoll," in Radioecology, Schultz, V., and Klement, A.W. Jr., Eds., Reinhold, New York, NY.

Held, E. E. (1965), Gamma Dose Rates at Rongelap Atoll, 1954-1963, University of Washington, Laboratory of Radiation Biology, Seattle, WA, UWFL-91.

Hisamatsu, S., and M. Sakanoue (1978), "Determination of Transuranium Elements in a So-Called "Bikini Ash" Sample and in Marine Sediment Samples Collected Near Bikini Atoll," Health Phys. 35, 301-307. 
Noshkin, V. E. (1980), “Transuranium Radionuclides in Components of the Benthic Environment of Enewetak Atoll," in Transuranic Elements in the Environment, Hanso, W. C., Ed., U.S. Department of Energy, Washington, DC, DOE/TIC-22800.

Noshkin, V. E., and K. M. Wong (1980), "Plutonium Mobilization from Sedimentary Sources to Solution in the Marine Environment," Marine Radioecology (Proc. 3rd NEA Seminar, Tokyo, 1979), NEA, OECD, Paris, p.729.

Noshkin, V. E., K. M. Wong, R. J. Eagle, and W. L. Robison (1987), “Comparative Concentrations of $137 \mathrm{Cs}$, $90 \mathrm{Sr}, 239,240 \mathrm{Pu}$ and 241Am in Tissues of Fish from the Marshall Islands and Calculated Dose Commitments from Their Consumption," in Environmental Research on Actinide Elements, Pinder, J .E. III, Alberts, J. J., McLeod, K. W., and R. G. Schreckhise, Eds., Proc. Symposium held at Hilton Head, SC, 1983, U.S. Department of Energy, Washington, D.C., CONF-841142.

Noshkin, V. E., K. M. Wong, R. J. Eagle, T. A. Jokela, and J. A. Brunk (1988), Radionuclide Concentrations in Fish and Invertebrates from Bikini Atoll, Lawrence Livermore National Laboratory, Livermore, CA, UCRL-53846.

Noshkin, V. E., R. J. Eagle, K. M. Wong, and W. L. Robison (1997a), Sediment Studies at Bikini Atoll Part II. Transuranium Elements in Surface Sediments, Lawrence Livermore National Laboratory, Livermore, CA, UCRL-LR-129379.

Noshkin, V. E., R. J. Eagle, J. L. Brunk, and W. L. Robison (1997b), Sediment Studies at Bikini Atoll Part II1. Inventories of Some Long Lived Gamma Emitting Radionuclides Associated with Lagoon Surface Sediment, Lawrence Livermore National Laboratory, Livermore, CA, UCRL-LR-129834.

Noshkin, V. E., R. J. Eagle, K. M. Wong, T. A. Jokela, and W. L. Robison (1981a), Radionuclide Concentrations and Dose Assessment of Cistern Water and Groundwater at the Marshall Islands, Lawrence Livermore National Laboratory, Livermore, CA, UCRL-52853 Pt. 2.
Noshkin, V. E., R. J. Eagle, K. M. Wong, T. A. Jokela, J. L. Brunk, and K. V. Marsh (1981b), Concentrations of Radionuclides in Reef and Lagoon Pelagic Fish from the Marshall Islands, Lawrence Livermore National Laboratory, Livermore, CA, UCID-19028.

Robison, W. L., and C. L. Conrado (1996a), Radiological Conditions at the Southern Islands of Rongelap Atoll, Lawrence Livermore National Laboratory, Livermore, CA, UCRL-ID-123375.

Robison, W. L. and C. L. Conrado (1996b), Radiological Conditions at Naen, Yugui, Lomiulal, Kabelle, and Mellu Islands in the Northern Half of Rongelap Atoll, Lawrence Livermore National Laboratory, Livermore, CA, UCRL-ID-123374.

Robison, W. L., C. L. Conrado, and K. T. Bogen (1994), An Updated Dose Assessment for Rongelap Island, Lawrence Livermore National Laboratory, Livermore, CA, UCRL-LR-107036.

Robison, W. L., V. E. Noshkin, C. L. Conrado, R. J. Eagle, J. L. Brunk, T. A. Jokela, M. E. Mount, W. A. Phillips, A. C. Stoker, M. L. Stuart, and K. M. Wong (1997), "The Northern Marshall Islands Radiological Survey: Data and Dose Assessments," Health Phys. 73, 37-48.

Robison, W. L., V. E. Noshkin, W. A. Phillips and R. J. Eagle (1981), The Northern Marshall Islands Radiological Survey: Radionuclide Concentrations in Fish and Clams and Estimated Doses Via the Marine Pathway, Lawrence Livermore National Laboratory, Livermore, CA, UCRL-52853 Pt. 3.

Wong, K. M., T. A. Jokela, and V .E. Noshkin (1994), Radiochemical Procedures for Analysis of $\mathrm{Pu}, \mathrm{Am}, \mathrm{Cs}$, and $\mathrm{Sr}$ in water, Soil, Sediments and Biota Samples, Lawrence Livermore National Laboratory, Livermore, CA, UCRL-ID-116497.

Wong, K. M., T. A. Jokela, and V. E. Noshkin (1980), "Problems Associated with Transuranium Determination of Suspended Soilids in Sea Water Samples," in Radioelement Analysis Progress and Problems, Lyon, W.S., Ed., Ann Arbor Press, Ann Arbor, MI. 


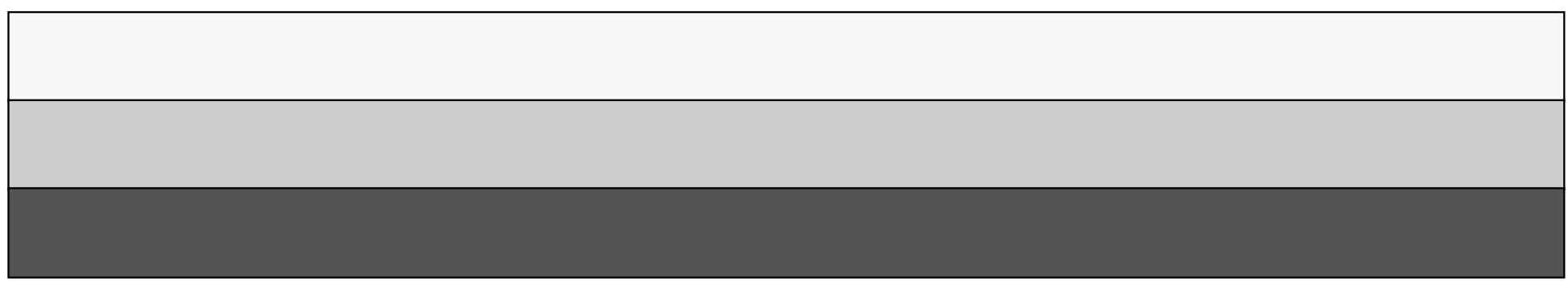

